\title{
Comparison of occurrence of intestinal parasites in children less than 16 years of age between rural and urban hospitals in Zambia in 2017
}

\begin{abstract}
Human intestinal parasitic infections are still endemic in rural and urban children in developing countries such as Zambia. We conducted a cross-sectional study to compare the occurrence of intestinal parasites in children less than 16 years between a rural hospital, Chilonga Mission Hospital CMH) in Mpika, and an urban hospital, Arthur Davison Hospital ADH) in Ndola, Zambia, in 2017. Hospital laboratory records of stool examinations results performed at both hospitals from January to December 2017 were analysed. The prevalence of intestinal parasites at CMH was $5.7 \%$ while that for ADH was $0.4 \%$. The difference between the two prevalences was statistically significant $(\mathrm{P}<0.005)$. One case of Balantidium coli, not previously known to be endemic in Africa, was detected at $\mathrm{CMH}$. We made recommendations on how to improve laboratory detection and control of intestinal parasites in children in endemic countries such as Zambia.
\end{abstract}

Volume 7 Issue 2 - 2018

\author{
Victor Mwanakasale,' Peter Songolo, ${ }^{2}$ \\ Jonathan Mwansa, ${ }^{3}$ Esther Musongole, ${ }^{3}$ \\ Elizabeth Mutamba, ${ }^{4}$ Amon Siame ${ }^{4}$ \\ 'Copperbelt University, School of Medicine, Zambi \\ ${ }^{2}$ World Health Organization, Country office, Zambia \\ ${ }^{3}$ Arthur Davison Children's Hospital, Zambia \\ ${ }^{4}$ Chilonga Mission Hospital, Zambia
}

Correspondence: Victor Mwanakasale, Senior Lecturer, Medical Parasitology, Copperbelt University, Ndola,

Zambia, Tel +260977804740, Email vickapasa@gmail.com

\section{Introduction}

Human Intestinal parasitic infections are prevalent globally mostly in developing countries. These infections cause high morbidity and mortality mostly in children. ${ }^{1}$ The parasites here are both helminthes and protozoans. The most common helminthic parasites are also known as geohelminths or Soil Transmitted Helminths (STHs). They include the following worms Ascaris lumbricoides (large intestinal round worms), Trichuris trichiura (whip worm), and hookworm (Ancylostoma duodenale and Necator americanus). It is estimated that A.lumbricoides infects over 1 billion people, while T.trichiura infects 795 million people, and hookworm infects 740 million people globally. ${ }^{2}$ These worms thrive in the tropics and subtropics of the developing world where environmental, socio-economic, demographic and health-related behavior influence their transmission and distribution. ${ }^{3}$ The most common protozoan parasites include Giardia lamblia, Entamoeba histolytica, Cyclospora cayetanensis, and Cryptosporidium spp. The infections caused by these parasites are associated with diarrhea. ${ }^{4}$ Among these infections amoebiasis is the third leading cause of mortality among parasitic diseases globally, affecting mostly developing countries. It is estimated that about 50 million people globally acquire invasive amoebic infection annually with 40-100 thousand deaths. ${ }^{5,6}$ Infection with G.lamblia is very common in developing world though it is also found in developed countries. Cryptosporidium spp infection occurs mostly among patients with immune deficiency such as Acquired Immune Deficiency Syndrome (AIDS) and children less than 5 years of age in both developed and developing countries. This parasite is transmitted mostly via faecal contamination as a result of poor sewage and poor quality water in developing countries.

Since the transmission of both intestinal protozoan parasites and helminthes in developing countries is influenced by common factors such as environmental, level of sanitation, level of personal hygiene, quality of water supply, and level of health education, geographical location of communities, being either rural or urban, will determine their distribution. Our study compared the occurrence of intestinal parasites in children less than 16 years of age in 2017 as detected during stool microscopy between a rural hospital and an urban hospitals in Zambia. The objectives of the study were to determine any differences and similarities in intestinal parasites detected during stool microscopy in children between an urban hospital, Arthur Davison Children's Hospital (ADH), Ndola city, Zambia, and a rural hospital, Chilonga Mission Hospital (CMH), Mpika, Zambia, in 2017, and come up with recommendations on how to improve the diagnosis and control of these parasites. We present our findings in this article.

\section{Methodology}

This was a cross-sectional study in which hospital laboratory records of microscopic stool examination results for the period from January to December 2017 were analysed for Arthur Davison Children's Hospital (ADH) and Chilonga Mission Hospital (CMH). At both hospitals stool samples were routinely examined for intestinal helminthes and protozoans using either wet mount light microscopy or the formal-ether concentration light microscopy methods while modified Zeehl-Neelsen stain light stool microscopy method was used for the detection of Cryptosporium spp. Clinical details as to the reasons for requesting stool examinations on the patients were not considered in this study.

\section{Results}

At ADH a total of 530 stool samples were examined while at $\mathrm{CMH}$ 194 stool samples were examined at CMH in the year. Two stool samples were positive for A.lumbricoides and Cryptosporidium spp at $\mathrm{ADH}$ while eleven stool samples were positive for A.lumbricoides, E.histolytica, G.lamblia, hookworm, Balantidium coli, and Strongyloides stercoralis at $\mathrm{CMH}$. This gave the prevalence of intestinal parasites at $\mathrm{ADH}$ of $0.4 \%$ while that of $\mathrm{CMH}$ was $5.7 \%$. There was a statistically significant difference between the two 
prevalences $(\mathrm{P}=0.00002)$. Table 1 below compares the frequency of occurrence of the individual parasites between the two hospitals. At $\mathrm{ADH}$ the two parasites detected were in children over five years of age. At $\mathrm{CMH}$ all the parasites detected were in children over five years of age apart from the two cases of G.lamblia which were detected in children 5 years and below.

Table I Frequency of occurrence of intestinal parasites by hospital and age of children

\begin{tabular}{llll}
\hline Hospital & $\begin{array}{l}\text { Age (years) } \\
\text { of Patient }\end{array}$ & Parasite Species & Frequency \\
\hline \multirow{2}{*}{ ADH } & 6 & Ascaris lumbricoides & I \\
& 14 & Cryptosporidium spp & I \\
& 6 & Ascaris lumbricoides & 1 \\
& 12 & Balantidium coli & I \\
& 6,11 & Entamoeba histolytica & 2 \\
$\mathrm{CMH}$ & 3,5 & Giardia lamblia & 2 \\
& $8,9,10,12$ & Hookworm & 4 \\
& 7 & Strongyloides stercoralis & 1 \\
\hline
\end{tabular}

\section{Discussion}

None of the stool samples at both hospitals detected STHs in children less than five years of age. This might imply that the twice per year deworming program by the Ministry of Health of Zambia through the Child Health week campaign for children less than five years of age is effective in keeping the children free of intestinal parasitic worms. During the child health week all children less than five years of age receive a dose of vitamin A and a deworming drug, either mebendazole $(500 \mathrm{mg}$ ) or albendazole $(400 \mathrm{mg})$. All the cases of STHs detected in both hospitals were in children over five years of age. Most likely these children were either out of school or they had missed the twice per year deworming exercise that is jointly conducted in primary schools by the Ministry of Health and Ministry of General Education of Zambia through the School Health month program. In the school health program, school children are given a single dose of either albendazole $(400 \mathrm{mg})$ or mebendazole $(500 \mathrm{mg})$ to clear STHs twice in a year. In schistosomiasis endemic areas, the program also includes once per year treatment of schistosomiasis with a single dose of praziquantel ( $40 \mathrm{mg} / \mathrm{kg}$ body weight). The more cases of STHs in rural children than in urban children observed here could be explained by the expected higher proportion of school age children out of school in rural areas than in urban areas.

At $\mathrm{CMH}$, two cases of E.histolytica infection were detected. However, this diagnosis couldn't be confirmed due to lack of confirmatory tests such as Isoenzyme analysis and Polymerase Chain Reaction (PCR). ${ }^{8}$ What was diagnosed as E.histolytica might have been Entamoeba dispar which has similar morphology to E.histolytica but is non-pathogenic. ${ }^{9,10}$ If the diagnosis of E.histolytica was true then the low prevalence recorded here is an underestimation of the true prevalence as stool microscopy used at CMH has low sensitivity in the diagnosis of E.histolytica. ${ }^{1}$ Several studies have demonstrated that E.histolytica-specific antigen detection test has high sensitivity and specificity for the detection of E.histolytica. ${ }^{11,12}$ Furthermore, there are several PCR-based tests that have been developed that are sensitive and specific for the detection of E.histolytica. ${ }^{8,13}$ Similarly the two cases of G.lamblia diagnosed at $\mathrm{CMH}$ is an underestimation of the true prevalence as stool microscopy has low sensitivity for the laboratory diagnosis of G.lamblia. More sensitive and specific diagnostic tests for the detection of G.lamblia such as G.lamblia specific antigen detection ELISA and PCR have been developed and are available commercially. ${ }^{14,15}$

What our study discovered of great interest was the one case of B.coli infection that was diagnosed at $\mathrm{CMH}$. Balantidiasis, infection caused by B.coli, is an uncommon zoonotic infection that has potential for worldwide distribution. ${ }^{16}$ The reservoir host for B.coli is the pig. Regions of high prevalence of B.coli in pigs include Latin America, the Philippines, Papua New Guinea, west Iran, and parts of the Middle East. ${ }^{17,18}$ It is therefore surprising that B.coli has been detected in Zambia. This therefore means that this infection may be endemic across Africa and goes undiagnosed because of either lack of experience by laboratory technicians examining the stools microscopically or the low sensitivity of single sample stool microscopy that is routinely performed in stool examination. ${ }^{19}$ Most cases of Balantidiasis in humans are asymptomatic just as observed in pigs and if symptoms do develop they may include dysentery.

\section{Conclusion}

Our study seems to suggest that children in rural areas of Zambia are more infected with intestinal parasites than children in urban areas. This might be true in other endemic countries in developing countries. However, it should be appreciated that collection of stool samples for microscopic examination of intestinal parasites in children less than five years of age is a challenge in most hospitals. A child might not be willing to void in the hospital environment when the doctor might want to send the stool sample to the laboratory for parasitological examination. Therefore the prevalence of intestinal parasites reported in this article may be a gross underestimation of the true prevalence of intestinal parasites in both rural and urban areas of Zambia.

\section{Recommendations}

We recommend that all out of school but school age children and all school children who miss deworming during school health month should be given two doses of deworming drugs in a year whenever they seek medical attention for any reason at both rural and urban hospitals in endemic countries such as Zambia. Contact amoebicides such Diloxamine furoate, Iodoquinol, and Paramomycin should be prescribed to rural children diagnosed with E.histolytica infection, in addition to 5-nitroimidazole compounds, to reduce contamination of the environment with cysts of the parasite and thereby reducing transmission of the parasite in endemic countries such as Zambia. Laboratory diagnostic tests with high sensitivity and specificity such Antigen detection tests and PCR-based tests should be introduced in rural and urban hospitals in endemic countries such as Zambia for the detection of E.histolytica, G.lamblia, and Cryptosporidium spp infections. In the laboratory detection of B.coli infection, multiple samples of stool for stool microscopy should be routinely done to improve on the sensitivity of this test. In addition, laboratory technicians in Africa should be made aware of the existence of B.coli in the region.

\section{Acknowledgement}

None. 


\section{Conflict of interest}

None.

\section{References}

1. Haque R. Huan Intestinal Parasites. J Jealth Popul Nutr. 2007;25(4):387391.

2. de Silva NR, Brooker S, Hotez PJ. Soil-transmitted helminth infections: updating the global picture. Trends Parasitol. 2003;19:547-51.

3. Norhayati M, Fatmah MS, Yusof S. Intestinal parasitic infections in man: a review. Med J Malaysia. 2003;58(2):296-305.

4. Davis AN, Haque R, Petri WA. Update on protozoan parasites of the intestine. Curr Opin Gastroentrol. 2002;18(1):10-4.

5. World Health Organization. Amoebiasis. WHO Weekly Epidemiol Rec. 1997;72:97-100.

6. Petri WA, Haque R, Lyerly D, et al. Estimating the impact of amoebiasis on health. Parasitol Today. 2000;16(8):320-21.

7. Okhuysen PC, White AC. Parasitic infections of the intestine. Curr Opin Infect Dis. 1999;12(5):467-72.

8. Haque R, Ali IK, Akther S, et al. Comparison of PCR, isoenzyme analysis, and antigen detection for diagnosis of Entamoeba histolytica infection. $J$ Clin Microbiol.1998;36(2):449-52.

9. Haque R, Huston $\mathrm{CD}$, Hughes $\mathrm{M}$, et al. Amoebiasis. $N$ Engl $\mathrm{J}$ Med. 2003;348:1565-73.

10. Haque R, Faruque AS, Hahn P, et al. Entamoeba histolytica and Entamoeba dispar infection in children in Bangladesh. J Infect Dis. 1997;175(3):7356.
11. Evangelopoulos A, Legakis N, Vakalis N. Microscopy, PCR, and ELISA applied to the epidemiology of amoebiasis in Greece. Parasitol Int. 2001;50(3):185-9.

12. Silva MC, Monteiro C do S, Araujo Bdos A, et al. Determination of Entamoeba histolytica infection in patients from Greater Metropolitan Belem, Para, Brazil, by enzyme-linked immunosorbent assay (ELISA) for antigen detection. Cad Saude Publica. 2005;21(3):969-73.

13. Roy S, Kabir M, Mondal D, et al. Real-time-PCR assay for diagnosis of Entamoeba histolytica infection. J Clin Microbiol. 2005;43(5):2168-72.

14. Aldeen WE, Carroll K, Robison A, et al. Comparison of nine commercially available enzyme-linked immunosorbent assays for detection of Giardia lamblia in fecal specimens. J Clin Microbiol. 1998;36(5):1338-40.

15. Ng CT, Gilchrist CA, Lane A, et al. Multiplex real-time PCR assay using Scorpion probes and DNA capture for genotype-specific detection of Giardia lamblia on fecal samples. J Clin Microbiol. 2005;43(3):1256-60.

16. Anargyrou KGL, Petrikkos MTE, Suller A, et al. Pulmonary Balantidium coli infection in a leukemic patient. Am J Hematol. 2003;73(3):180-183.

17. Solaymani-Mohammadi S, Rezaian M, Hooshyar H, et al. Intestinal protozoa in wild boars (Sus scrofa) in Western Iran. $J$ Wildl Dis. 2004;40(4):801-803.

18. Yazar S, Altuntas F, Sahin I. Dysentery caused by Balantidium coli in a patient with non-Hodgkin's lymphoma from Turkey. World J Gastroenterol. 2004;10(3):458-459.

19. Garcia LS. Diagnostic medical parasitology. $5^{\text {th }}$ ed. ASM Press, Washington DC. 2007. 